\title{
Long-term outcomes of immediate autologous breast reconstruction after definite adjuvant therapy in intermediate and locally advanced breast cancer
}

\author{
Won Sup Yoon ${ }^{1}$, Chai Hong Rim ${ }^{1}$, Dae Sik Yang ${ }^{2}$, Jung Ae Lee ${ }^{2}$, Gil Soo Son ${ }^{3}$, Young Woo Chang ${ }^{3}$, \\ Sang Uk Woo ${ }^{4}$, Deok-Woo Kim ${ }^{5}$, Eun-Sang Dhong ${ }^{6}$
}

${ }^{1}$ Department of Radiation Oncology, Ansan Hospital, Korea University Medical Center, Ansan, Republic of Korea; ${ }^{2}$ Department of Radiation Oncology, Guro Hospital, Korea University Medical Center, Seoul, Republic of Korea; ${ }^{3}$ Department of Surgery, Ansan Hospital, Korea University Medical Center, Ansan, Republic of Korea; ${ }^{4}$ Department of Surgery, Guro Hospital, Korea University Medical Center, Seoul, Republic of Korea; ${ }^{5}$ Department of Plastic Surgery, Ansan Hospital, Korea University Medical Center, Ansan, Republic of Korea; ${ }^{6}$ Department of Plastic Surgery, Guro Hospital, Korea University Medical Center, Ansan, Republic of Korea

Contributions: (I) Conception and design: DS Yang, GS Son, WS Yoon; (II) Administrative support: CH Rim, JA Lee; (III) Provision of study materials or patients: GS Son, YW Chang, SU Woo, DW Kim, ES Dhong, WS Yoon, DS Yang; (IV) Collection and assembly of data: WS Yoon, CH Rim, JA Lee; (V) Data analysis and interpretation: WS Yoon, CH Rim, JA Lee; (VI) Manuscript writing: All authors; (VII) Final approval of manuscript: All authors.

Correspondence to: Dae Sik Yang, MD, PhD. Department of Radiation Oncology, Guro Hospital, Korea University Medical Center, 148 Gurodongro, Seoul 08308, Republic of Korea. Email: irionmphage@korea.ac.kr; Gil Soo Son, MD, PhD. Department of Surgery, Ansan Hospital, College of Medicine, Korea University, 123 Jeokgeumro, Ansan 15355, Republic of Korea. Email: gsson@korea.ac.kr.

Background: To analyze oncologic outcomes and reconstruction quality in locally advanced stage breast cancer after performing immediate autologous breast reconstruction (IABR).

Methods: From 2007 to 2014, data of patients aged $\leq 70$ years old with stage II-III breast cancer who received total mastectomy (TM) were extracted from medical records. Exclusion criteria were: previous contralateral breast cancer, follow-up loss before adjuvant therapy completion, and artificial reconstruction. Patients were divided into two groups: (I) TM alone, and (II) TM + IABR. Overall survival (OS) and locoregional recurrence free survival (LRRFS) were calculated. Times of minor revision, abnormal image findings in breast, and change of breast height were observed.

Results: Sixty-one of 188 patients received IABR after TM. Stage IIIB-C was the most important prognostic factor for OS $(\mathrm{P}<0.001)$ and LRRFS $(\mathrm{P}<0.001)$. For stage II, five-year TM and TM + IABR OS rates were $96.8 \%$ and $100 \%(\mathrm{P}=0.324)$, respectively. For stage IIIB-C, five-year TM and TM + IABR OS rates were $57.6 \%$ and $62.5 \%(\mathrm{P}=0.544)$, respectively. For stage II, five-year TM and TM + IABR LRRFS were $98.1 \%$ and $95.7 \%(\mathrm{P}=0.998)$, respectively. For stage IIIB-C, five-year TM and TM + IABR LRRFS were $70.8 \%$ and $62.5 \%(\mathrm{P}=0.378)$, respectively. Two major complications were observed after IABR. Minor revisions, abnormal image findings, and change of breast height were common without showing significant relation with adjuvant radiotherapy or tumor stage.

Conclusions: IABR showed feasible oncologic outcomes in 5-year follow-up. Adjuvant radiotherapy had little effect on quality of reconstruction. However, IABR in advanced stage should be cautiously applied considering expected survival and minor problems after IABR.

Keywords: Breast cancer; mastectomy; radiotherapy; reconstruction; survival

Submitted Aug 14, 2019. Accepted for publication Nov 14, 2019.

doi: 10.21037/atm.2019.11.129

View this article at: http://dx.doi.org/10.21037/atm.2019.11.129 


\section{Introduction}

Breast cancer is common in women worldwide. According to 2013 data, one in 18 women developed breast cancer until 79 years, with 1.8 million incident cases of breast cancer and 464,000 deaths globally (1). In Korea between 2001 and 2012, 10-year overall survivals (OS) of stages I, II, III, and IV breast cancer were $92.7 \%, 84.8 \%, 63.4 \%$, and $22.2 \%$, respectively (2). In the Netherlands, contemporary groups from 2006 to 2012 had better OS than those from 1999 to 2005 because various effective medicines have been developed (3). Survivorship including breast preservation and quality of life are currently rising issues in the management of breast cancer survivals.

Breast conserving surgery was done in $67 \%$ of overall surgical procedures in Korea from 2012 to 2013 (4). However, mastectomy rate has arisen slightly since 2014 because the widespread use of preoperative MRI that can accurately detect tumor extent and genetic tests that can increase prophylactic mastectomy. Breast reconstruction is another option after mastectomy to preserve breast shape. It has been commonly done in early breast cancer or prophylactic mastectomy. However, some cases need unexpected adjuvant radiotherapy and chemotherapy due to discordance between clinical and pathologic stages. Besides, some patients desire breast reconstruction after mastectomy in spite of their advanced stage.

Immediate autologous breast reconstruction (IABR) is one of reconstruction methods using a patient's own tissue. A meta-analysis has shown that reconstructive failure and surgical-site infection are lower in IABR than those in reconstructions by tissue expander or implant (5). Another meta-analysis has reported that IABR has less morbidity than implants in the setting of adjuvant radiotherapy, although radiotherapy can increase overall risks of morbidity after breast reconstruction (6). In addition, patient-reported outcomes of IABR are superior to those of implants (7). Therefore, IABR could be highly recommended for advanced stage breast cancer patients who want breast reconstruction. Although clinical safety of IABR has been well-established in early stage breast cancer, the clinical effectiveness of IABR for locally advanced stage that needs more intensive chemotherapy and radiotherapy has been rarely presented. Thus, the objective of this study was to analyze oncologic outcomes and reconstruction quality in locally advanced stage breast cancer after performing IABR.

\section{Methods}

Patient data from two institutions were included in this retrospective study. Patients from January 2007 to December 2014 in one institution and from January 2013 to December 2014 in the other institution reflecting the general implementation of IABR procedure were included. Eligible criteria were: (I) those who were pathologically or clinically [if neoadjuvant chemotherapy (NACT) was done] confirmed intermediate or locally advanced breast cancer (stage II-III of AJCC 7th edition); (II) age $\leq 70$ years; (III) definite surgical resection ( $\mathrm{R} 0$ or $\mathrm{R} 1$ resection) of total mastectomy (TM). Exclusive criteria were: (I) those who had artificial or delayed breast reconstruction; (II) insufficient follow-up information before completion of adjuvant chemotherapy or radiotherapy; (III) previous breast cancer history of carcinoma in situ or invasive carcinoma; (IV) other malignancies except early stage thyroid cancer or squamous skin cancer. Institutional Review Board (IRB) of our hospital approved this study (IRB No. K2018-1002) with an informed consent waiver.

The possibility of IABR was sufficiently explained to patients who needed TM by oncologic and plastic surgeons. After discussing various topics related to the expected effect and sequelae, IABR was performed after patients agreed to receive it. According to pathologic outcomes and molecular findings, adjuvant therapies such as chemotherapy, radiotherapy, hormonal therapy, and target therapy were determined based on boundaries of universal guidelines for breast cancer and national insurance policy of actual treatment period. For some advanced stage cases, NACT was tried before TM.

Personal and clinical information was acquired through medical record review. Because patients with NACT were included in this study, clinical staging of the 7 th edition AJCC was applied. Pathologic staging was applied to other patients. Molecular subtype was determined using the 8 th edition AJCC guidelines. In cases with two positives for HER-2 receptor in immunohistochemistry tests without in situ hybridization test, the group with histologic grade 3 was referred to as a positive finding while grade 1 or 2 was considered a negative finding.

For primary endpoints of oncologic outcomes, OS and loco-regional recurrence free survival (LRRFS) were calculated and compared between the TM group and the $\mathrm{TM}+\mathrm{IABR}$ group. As secondary endpoints, disease 
free survival (DFS) and distant metastases free survival (DMFS) were calculated. Survival observation started on the date of initial biopsy and finished on the date of specific events or the last follow-up. Locoregional event included ipsilateral chest wall and regional lymph nodes. Distant metastases included contralateral breast and regional nodes and metastases to other organs. For cases with adjuvant chemotherapy, the time-to-chemotherapy (TTC) between TM and the first chemotherapy was calculated as another secondary endpoint.

For morbidities of IABR, major complications and minor surgical revisions were reviewed. Complete flap failure or destruction of reconstruction over half was regarded as a major complication while other revisions after 3 months of IABR such as excision of focal fat necrosis, correction of asymmetry, and so on were regarded as minor revisions, including cases of major complications. Abnormal image findings of fat necrosis, seroma, and adhesive mass on CT were sequentially observed at about 6,18 , and 42 months after IABR. In addition, breast height change was observed on CT. Breast height was defined as maximum distance from chest wall to breast skin. Three breast heights of central axis (contralateral nipple level) and $4 \mathrm{~cm}$ upper and 4 $\mathrm{cm}$ lower axes from central axis were summed. A reduction of $10 \%$ during the observation period was regarded as positive finding.

Chi-square test was performed to evaluate TM and TM + IABR. Survival was calculated using Kaplan-Meier method. Survival difference in terms of risk factors was compared using log-rank test. Prognostic factors with a $\mathrm{P}$ value less than 0.1 in univariate analyses and a default of treatment group were entered into Cox-regression multivariate analyses using step-wise method. T-test was done to compare TTC and volume change of abnormal image findings. Chi-square test was done for minor revisions and height changes in terms of additional therapeutic methods and tumor stage. SPSS 20.0 (IBM SPSS Inc., Chicago, IL, USA) was used for all statistical analyses. Two-sided P values of less than 0.05 were considered significant.

\section{Results}

IABR was done in 61 of 188 patients with TM. NACT and postoperative radiotherapy (PORT) were done in 27 and 80 patients, respectively. Median age of the TM + IABR group was 43 years (range, 28-67 years). It was 50 years (range, 31-70 years) for the TM group. Stage II, IIIA, and IIIB-C patients accounted for $59.0 \%, 19.1 \%$, and $21.8 \%$, respectively. The rate of stage IIIB-C in TM was slightly higher than that in TM + IABR $(26.0 \%$ vs. $13.1 \%, \mathrm{P}=0.078)$. For TM + IABR, PORT was done in $37.7 \%$ (23/61) of overall patients and $71.4 \%(15 / 21)$ of stage III patients (Table 1). Transverse rectus myocutaneous flap was usually done except that latissimus dorsi flap was done for 5 patients.

Nineteen loco-regional recurrences (including 8 chest wall recurrences), 28 distant metastases, and 20 deaths were observed during a median follow-up of 56.8 months. In univariate analyses, T-stage, $\mathrm{N}$-stage, tumor stage, surgical margin, lymphovascular invasion, molecular subtype, and histologic grade were significantly related to OS (Table 2). In multivariate analyses, tumor stage was the most significant prognostic factor for both OS (stage IIIB-C, HR: $11.18,95 \%$ CI: $3.15-39.65, \mathrm{P}<0.001)$ and LRRFS (stage IIIB-C, HR: 12.07, 95\% CI: 4.27-34.09, P<0.001). In addition, T-stage (T4, HR: 2.60, 95\% CI: 0.94-7.19, $\mathrm{P}=0.065)$ was prognostic factor for OS and molecular subtype (basal type, HR: 3.12, 95\% CI: 1.08-9.00, $\mathrm{P}=0.036$ ) was prognostic factor for LRRFS. Five-year OS rates in TM and TM + IABR groups were $96.8 \%$ and $100 \%$ $(\mathrm{P}=0.324)$ for stage II, $95.5 \%$ and $91.7 \%(\mathrm{P}=0.698)$ for stage IIIA, and $57.6 \%$ and $62.5 \%(\mathrm{P}=0.544)$ for stage IIIB-C, respectively (Figure 1). Five-year LRRFS in TM and TM + IABR groups were $98.1 \%$ and $95.7 \%(\mathrm{P}=0.998)$ for stage II, $91.1 \%$ and $100 \%(\mathrm{P}=0.277)$ for stage IIIA, and $70.8 \%$ and $62.5 \%(\mathrm{P}=0.378)$ for stage IIIB-C, respectively. However, two locoregional failures were developed in stage IIIB-C of TM + IABR at 71 and 94 months (Figure 2). For TTC, $\mathrm{TM}+$ IABR (median 35.5 days) was relatively longer than TM (median 25 days) (mean difference: 10.7 days, 95 \% CI: 7.9-13.4 days, $\mathrm{P}<0.001)$.

Two major complications were observed. One was immediately after IABR and the other was after five months of PORT due to aggravation of fat necrosis. Minor revisions at three months after IABR, including two major complications after IABR, were done in $49.2 \%$ of patients. Radiologic evaluations were possible in $85.2 \%$ between 6 and 18 months (early-phase observation) and in $77.1 \%$ between 6 and 42 months (middle-phase observation). The reduction of breast height was $21.2 \%(11 / 52)$ and $31.9 \%$ $(15 / 47)$ in early-phase and middle-phase observations, respectively. The volume of abnormal imaging was over 10 cc in $27.3 \%(15 / 55)$ on CT at 6 months after IABR. The volume of abnormal imaging was well regressed, with a mean regression of $38.3 \%$ (SE: $5.1 \%$ ) and $59.3 \%$ (SE: $4.1 \%$ ) in early-phase and middle-phase observations, respectively. Minor revision, reduction of breast height, or volume 
Table 1 Patients characteristics

\begin{tabular}{|c|c|c|c|}
\hline Characteristics & $\mathrm{TM}(\mathrm{N}=127)$ & $\mathrm{TM}+\mathrm{IABR}(\mathrm{N}=61)$ & $P$ value \\
\hline$\leq 50:>50$ & 65:62 & $45: 16$ & 0.002 \\
\hline Laterality (right:left) & $60: 67$ & $32: 29$ & 0.536 \\
\hline Multiplicity (single:multiple) & $53: 74$ & $26: 35$ & 1.000 \\
\hline Histologic Grade (1:2:3) & $17: 62: 48$ & $8: 36: 17$ & 0.348 \\
\hline Molecular type (Lum-A:Lum-B:HER-2:Basal) & $44: 15: 49: 19$ & $28: 5: 24: 4$ & 0.110 \\
\hline T-stage (T1:T2:T3:T4) & $20: 72: 19: 16$ & $10: 43: 5: 3$ & 0.073 \\
\hline $\mathrm{N}$-stage (N0:N1:N2:N3) & $24: 61: 22: 20$ & $15: 28: 11: 7$ & 0.359 \\
\hline Lymphovascular invasion (no:yes) & $73: 54$ & $36: 24^{*}$ & 0.874 \\
\hline Chemotherapy (no:yes) & $5: 122$ & $2: 59$ & 1.000 \\
\hline Neoadjuvant & 23 & 5 & 0.083 \\
\hline Adjuvant & 116 & 58 & 0.554 \\
\hline Taxane & 95 & 45 & 0.860 \\
\hline Radiotherapy (no:yes) & $70: 57$ & $38: 23$ & 0.431 \\
\hline Stage II:IIIA:IIIB-C (in yes) & $18: 15: 24$ & $8: 8: 7$ & 0.078 \\
\hline Hormonal therapy (no:yes) & $39: 88$ & $14: 47$ & 0.302 \\
\hline
\end{tabular}

*, missing data. TM, total mastectomy; IABR, immediate autologous breast reconstruction; IDC, invasive ductal carcinoma.

Table 2 Comparison of survival in terms of prognostic factors

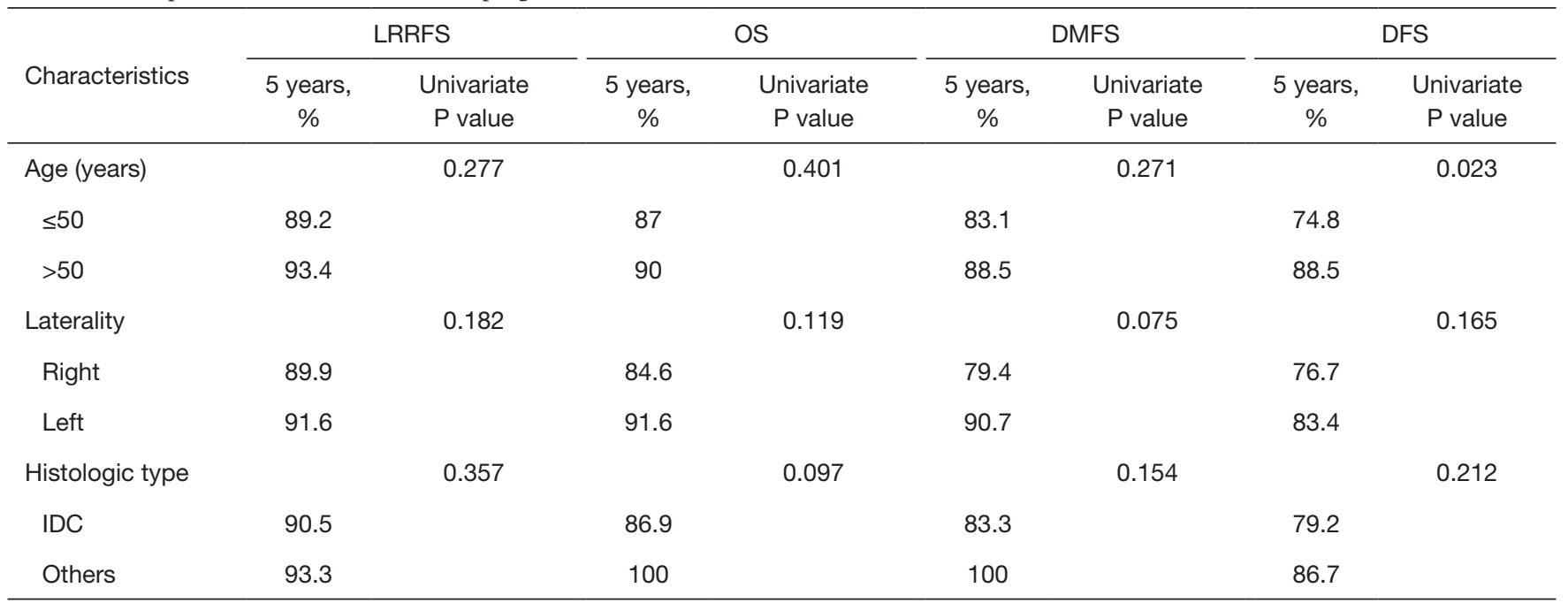

Table 2 (continued) 
Table 2 (continued)

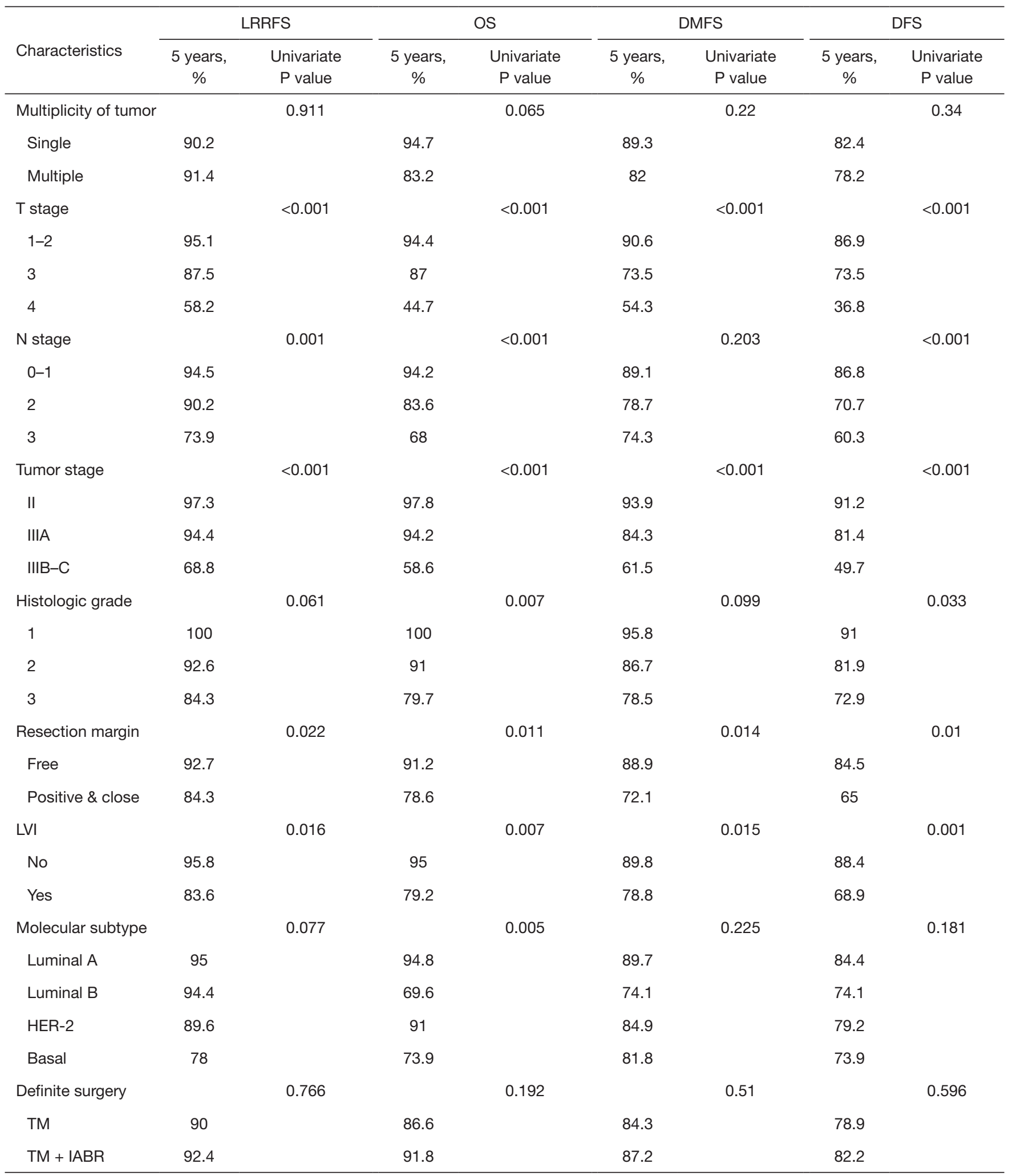

LRRFS, locoregional recurrence free survival; OS, overall survival; DMFS, distant metastases free survival; DFS, disease free survival; IDC, invasive ductal carcinoma; LVI, lymphovascular invasion; TM, total mastectomy; IABR, immediate autologous breast reconstruction. 

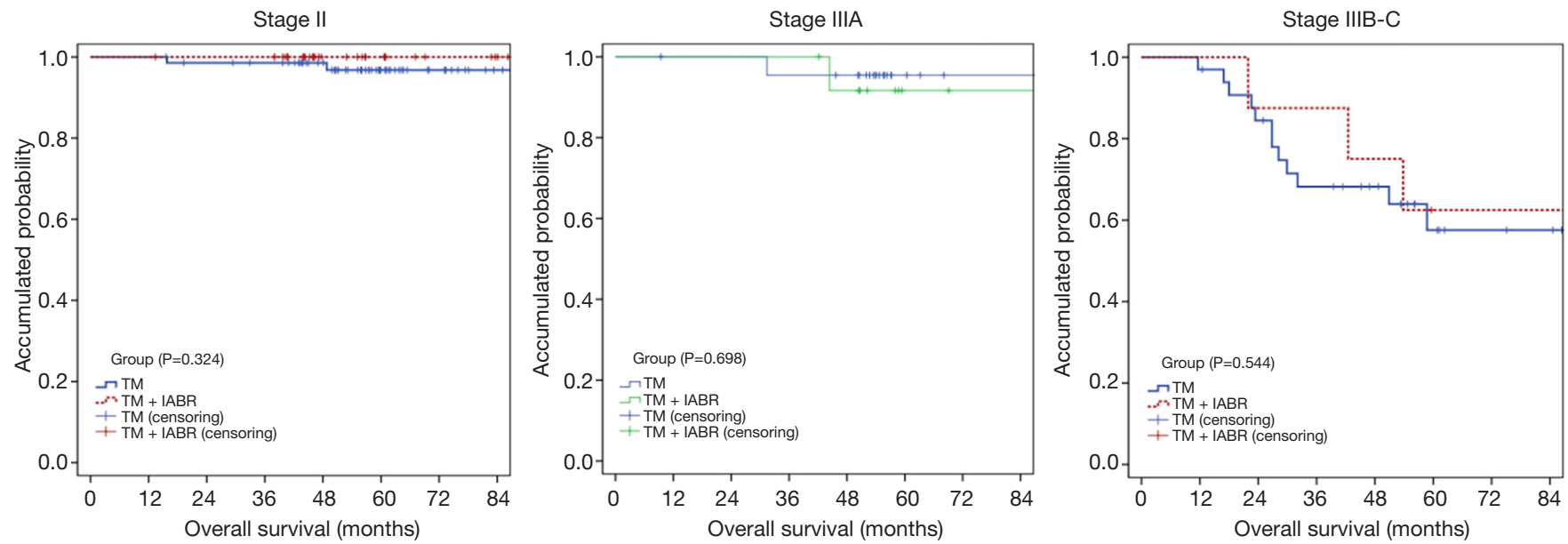

Figure 1 Comparisons of overall survival between TM and TM + IABR. (A) Stage II; (B) stage IIIA; and (C) stage IIIB-C. TM, total mastectomy; IABR, immediate autologous breast reconstruction.
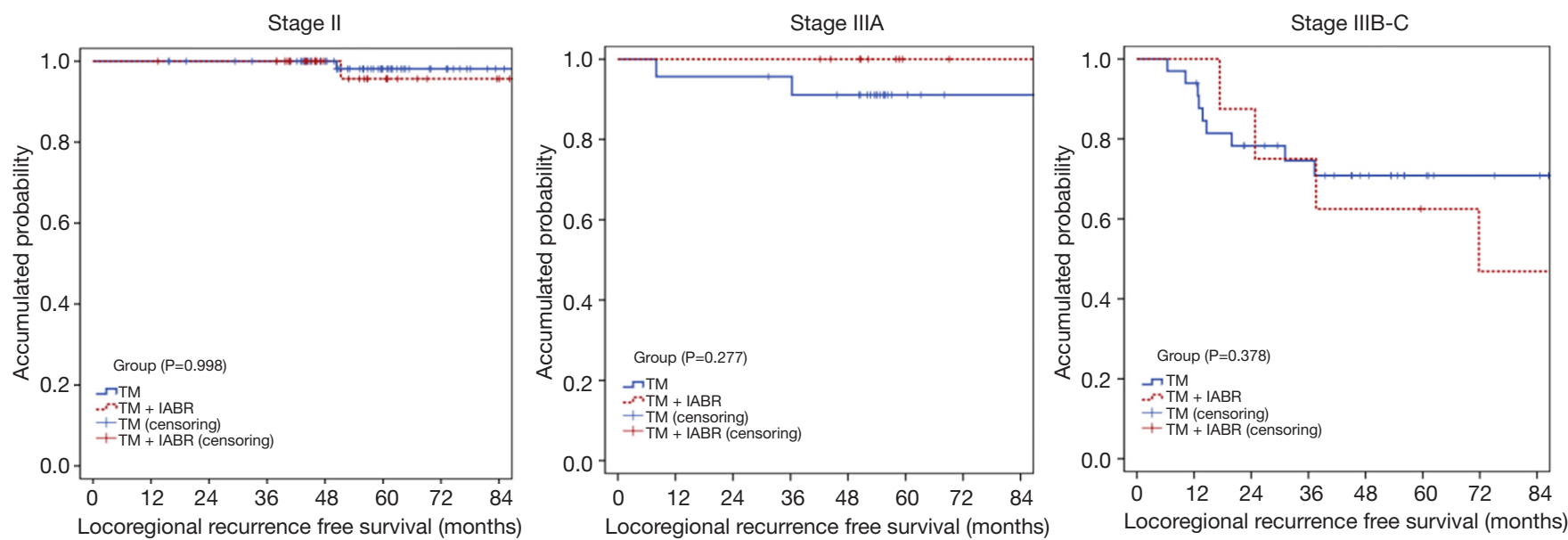

Figure 2 Comparisons of locoregional recurrence free survival between TM and TM + IABR. (A) Stage II; (B) stage IIIA; and (C) stage IIIB-C. TM, total mastectomy; IABR, immediate autologous breast reconstruction.

change of abnormal imaging was not related to therapeutic methods, including PORT or tumor stage (Table 3).

\section{Discussion}

We compared 188 patients receiving $\mathrm{TM}$ or $\mathrm{TM}+$ IABR in intermediate and locally advanced stage breast cancer. Tumor stage was the most important risk factor in multivariate analyses. For stage II, good oncologic outcomes were presented, with 5-year OS, DFS, and DMFS over $90 \%$ and 5-year LRRFS reaching up to $97.3 \%$. While limited survival deteriorations of about $3-10 \%$ occurred in stage IIIA, poor oncologic outcomes $(68.8 \%$ of 5 -year OS and $58.6 \%$ of 5-year LRRFS) were presented in stage IIIB-C. Although additional IABR after TM did not aggravate oncologic outcomes in stage IIIB-C, the LRRFS rate was high and the possibility of salvage therapeutics after standard therapies could be high in locally advanced stage. This situation could diminish subjective satisfaction of body images after IABR. In a French single institutional study (stage IIIA 28.8\%), 10-year OS and DFS after NACT and preoperative radiotherapy were $66.7 \%$ and $59.0 \%$, respectively, with a total local recurrence of $2.2 \%(8)$. A Japanese long-term observation over 5 -year showed that 
Table 3 Minor revisions and breast height reduction of immediate autologous breast reconstruction in terms of therapeutic methods and tumor stage

\begin{tabular}{|c|c|c|c|c|c|c|c|c|c|}
\hline \multirow{2}{*}{ Characteristics } & \multicolumn{3}{|c|}{ Minor revisions $(\mathrm{N}=61)$} & \multicolumn{3}{|c|}{$\begin{array}{l}\text { Reduction of breast height in early } \\
\text { phase }(\mathrm{N}=52)\end{array}$} & \multicolumn{3}{|c|}{$\begin{array}{l}\text { Reduction of breast height in } \\
\text { middle phase }(\mathrm{N}=47)\end{array}$} \\
\hline & No & Yes & $P$ value & No & Yes & $P$ value & No & Yes & $P$ value \\
\hline No & 21 & 17 & & 25 & 6 & & 23 & 8 & \\
\hline No & 29 & 27 & & 38 & 9 & & 29 & 14 & \\
\hline Yes & 2 & 3 & & 3 & 2 & & 3 & 1 & \\
\hline Taxane chemotherapy & & & 0.939 & & & 0.556 & & & 0.552 \\
\hline No & 5 & 9 & & 11 & 1 & & 8 & 3 & \\
\hline Yes & 26 & 21 & & 30 & 10 & & 24 & 12 & \\
\hline Target therapy & & & 0.613 & & & 0.897 & & & 0.552 \\
\hline No & 22 & 23 & & 29 & 8 & & 24 & 10 & \\
\hline Yes & 9 & 7 & & 12 & 3 & & 8 & 5 & \\
\hline Stage & & & 0.717 & & & 0.217 & & & 0.465 \\
\hline II & 21 & 19 & & 27 & 14 & & 20 & 11 & \\
\hline
\end{tabular}

disease recurrence was $4 / 10$ and death by breast cancer was $3 / 10$ in stage III after IABR and PORT (9). Of 22 patients with locally advanced cancer receiving NACT and preoperative radiotherapy with immediate reconstruction in a German report, 2-year OS and 2-year LRRFS were $89.3 \%$ and $95.2 \%$, respectively (10). In a Korean single institutional study including 75 cases of stage IIIA among 1000 IABR cases, there were only 3 cases of local recurrence in stage IIIA with insignificant influence of local recurrence on OS (11). There was no statistical difference in OS or LRRFS between TM and immediate breast reconstruction in another Korean case-control study including 6.2\% of stage III (12). Our study was unique in that it showed comparison outcomes of OS, DFS, DMFS, and LRRFS for TM + IABR $v s$. TM according to tumor stage because most reports showed single-group outcomes or focused on local recurrence or surgical methods.

Of our secondary end-point, TTC was delayed in TM
+ IABR. The National database from the United States of America showed that IABR was a risk factor for a delay of adjuvant chemotherapy (13). A large-scale study has emphasized the delay of adjuvant chemotherapy beyond 8 weeks can negatively affect DFS in a comparison group within 4 weeks in terms of all molecular subtypes except luminal-A type (14). Therefore, it is more important to reduce postoperative complications and recover performance status in advanced stage with IABR.

Regarding reconstruction quality after radiotherapy, several studies have reported that morbidities of fat necrosis and volume loss are increased. Mirzabeigi et al. have reported that fat necrosis and volume loss of IABR are increased by about 5 and 6 times, respectively, after PORT (15). Garvey et al. have reported that PORT can increase fat necrosis after IABR (22.5\% vs. 9.2\%) without showing difference in overall complication rate (16). Another database study has shown that PORT can increase infection 
rates beyond 6 months of radiotherapy (17). However, subjective reports by patients after IABR and PORT are not inferior in comparison with non-irradiated or delayed reconstruction groups (18-20). Except for one case of major complication, new lesion or continuous progression of abnormal image finding was not observed in our 23 patients with IABR plus radiotherapy. Volumes of these lesions continuously reduced and remained calcified regardless of radiotherapy or other therapeutic methods. Breast flap contracture was roughly measured by breast height change in our study. It might have progressed with the passage of time. In patients with locally advanced stage, because repeated minor revisions could cause more severe anxiety than early stage and negatively influence subjective oriented assessments, it should be sufficiently explained to patients before IABR.

Most of our patients received chemotherapy, including five patients with NACT in TM + IABR group. Therapeutic methods showed no influence on the quality of reconstruction in our study. Of 247 patients receiving chemotherapy and immediate breast reconstruction, there was no significant difference in infection rate according to chemotherapy timing or reconstruction method in a US study (21). In other small-sized studies, there were no significant differences in complications or wound infections receiving IABR after NACT $(22,23)$. In addition, two studies have analyzed immediate breast reconstruction after NACT and preoperative radiotherapy and found that the morbidity rate is acceptable $(24,25)$.

In our study, all tumor recurrences occurred in the chest wall. It is anticipated that tumor contamination of the central flap is rare. Our previous study suggested a technique to spare the central flap area for reducing breast contracture after PORT (26). Implant reconstruction does not compromise the technique quality of radiotherapy in comparison with TM except cases including internalmammary lymph node that increase mean and maximum heart doses in the implant reconstruction group (27). A customized bolus was suggested to reduce skin fibrosis, volume loss and contour deformities by minimizing radiation dose to internal-mammary vessels, anastomoses and skin (28).

Although our study was of middle size in scale for IABR and radiotherapy, it had some limitations. First, the followup period was not long enough because locoregional recurrences and distant metastases could persistently happen after five years of observation in breast cancer. Therefore, continuous follow-up is needed. Second, measurement methods such as breast height and abnormal volume findings were not absolute or systematic because periodic examination was done for tumor evaluation, not for examining the quality of reconstruction. Last, the sample size of stage III patients was too small to have sufficient statistical power. Therefore, caution should be exercised when interpreting our results. Another systematic review gathering these groups is warranted to clarify their oncologic outcomes.

\section{Conclusions}

With a close to five years of follow-up, TM + IABR did not aggravate oncologic outcomes in comparison with TM. PORT did not deteriorate quality of reconstruction regarding the possibility of minor revisions, breast height change, or abnormal image findings. For stage II cancer, IABR could be a feasible option for patients who want to preserve body image. However, it should be kept in mind that absolute oncologic outcomes of advanced stage might not be sufficient as common locoregional recurrences and minor problems could frequently develop after IABR.

\section{Acknowledgments}

Funding: This work was supported by Korea University [grant numbers K1912721].

\section{Footnote}

Conflicts of Interest: The authors have no conflicts of interest to declare.

Ethical Statement: The authors are accountable for all aspects of the work in ensuring that questions related to the accuracy or integrity of any part of the work are appropriately investigated and resolved. Institutional Review Board of Ansan Hospital approved this study (IRB No. K2018-1002) with an informed consent waiver.

\section{References}

1. Global Burden of Disease Cancer Collaboration, Fitzmaurice C, Dicker D, et al. The Global Burden of Cancer 2013. JAMA Oncol 2015;1:505-27.

2. Park EH, Min SY, Kim Z, et al. Basic Facts of Breast Cancer in Korea in 2014: The 10-Year Overall Survival Progress. J Breast Cancer 2017;20:1-11. 
3. Saadatmand S, Bretveld R, Siesling S, et al. Influence of tumour stage at breast cancer detection on survival in modern times: population based study in 173,797 patients. BMJ 2015;351:h4901.

4. Kang SY, Kim YS, Kim Z, et al. Basic Findings Regarding Breast Cancer in Korea in 2015: Data from a Breast Cancer Registry. J Breast Cancer 2018;21:1-10.

5. Tsoi B, Ziolkowski NI, Thoma A, et al. Safety of tissue expander/implant versus autologous abdominal tissue breast reconstruction in postmastectomy breast cancer patients: a systematic review and meta-analysis. Plast Reconstr Surg 2014;133:234-49.

6. Barry M, Kell MR. Radiotherapy and breast reconstruction: a meta-analysis. Breast Cancer Res Treat 2011;127:15-22.

7. Jagsi R, Momoh AO, Qi J, et al. Impact of Radiotherapy on Complications and Patient-Reported Outcomes After Breast Reconstruction. J Natl Cancer Inst 2018;110:157-65.

8. Monrigal E, Dauplat J, Gimbergues P, et al. Mastectomy with immediate breast reconstruction after neoadjuvant chemotherapy and radiation therapy. A new option for patients with operable invasive breast cancer. Results of a 20 years single institution study. Eur J Surg Oncol 2011;37:864-70.

9. Terao Y, Taniguchi K, Fujii M, et al. Postmastectomy radiation therapy and breast reconstruction with autologous tissue. Breast Cancer 2017;24:505-10.

10. Pazos M, Corradini S, Dian D, et al. Neoadjuvant radiotherapy followed by mastectomy and immediate breast reconstruction: An alternative treatment option for locally advanced breast cancer. Strahlenther Onkol 2017;193:324-31.

11. Lee TJ, Hur WJ, Kim EK, et al. Outcome of management of local recurrence after immediate transverse rectus abdominis myocutaneous flap breast reconstruction. Arch Plast Surg 2012;39:376-83.

12. Ryu JM, Paik HJ, Park S, et al. Oncologic Outcomes after Immediate Breast Reconstruction Following Total Mastectomy in Patients with Breast Cancer: A Matched Case-Control Study. J Breast Cancer 2017;20:74-81.

13. Riba LA, Gruner RA, Fleishman A, et al. Surgical Risk Factors for the Delayed Initiation of Adjuvant Chemotherapy in Breast Cancer. Ann Surg Oncol 2018;25:1904-11.

14. Yu KD, Fan L, Qiu LX, et al. Influence of delayed initiation of adjuvant chemotherapy on breast cancer survival is subtype-dependent. Oncotarget 2017;8:46549-56.
15. Mirzabeigi MN, Smartt JM, Nelson JA, et al. An assessment of the risks and benefits of immediate autologous breast reconstruction in patients undergoing postmastectomy radiation therapy. Ann Plast Surg 2013;71:149-55.

16. Garvey PB, Clemens MW, Hoy AE, et al. Muscle-sparing TRAM flap does not protect breast reconstruction from postmastectomy radiation damage compared with the DIEP flap. Plast Reconstr Surg 2014;133:223-33.

17. Jagsi R, Jiang J, Momoh AO, et al. Complications After Mastectomy and Immediate Breast Reconstruction for Breast Cancer: A Claims-Based Analysis. Ann Surg 2016;263:219-27.

18. Berthet G, Faure C, Dammacco MA, et al. Tolerance of latissimus dorsi in immediate breast reconstruction without implant to radiotherapy. J Plast Reconstr Aesthet Surg 2018;71:15-20.

19. Billig J, Jagsi R, Qi J, et al. Should Immediate Autologous Breast Reconstruction Be Considered in Women Who Require Postmastectomy Radiation Therapy? A Prospective Analysis of Outcomes. Plast Reconstr Surg 2017;139:1279-88.

20. Steele KH, Macmillan RD, Ball GR, et al. Multicentre study of patient-reported and clinical outcomes following immediate and delayed Autologous Breast Reconstruction And Radiotherapy (ABRAR study). J Plast Reconstr Aesthet Surg 2018;71:185-93.

21. Chattha AS, Cohen JB, Bucknor A, et al. Surgical site infection in immediate breast reconstruction: Does chemotherapy timing make a difference? J Surg Oncol 2018;117:1440-6.

22. Schaverien MV, Munnoch DA. Effect of neoadjuvant chemotherapy on outcomes of immediate free autologous breast reconstruction. Eur J Surg Oncol 2013;39:430-6.

23. Tanaka S, Hayek G, Jayapratap P, et al. The Impact of Chemotherapy on Complications Associated with Mastectomy and Immediate Autologous Tissue Reconstruction. Am Surg 2016;82:713-7.

24. Paillocher N, Florczak AS, Richard M, et al. Evaluation of mastectomy with immediate autologous latissimus dorsi breast reconstruction following neoadjuvant chemotherapy and radiation therapy: A single institution study of 111 cases of invasive breast carcinoma. Eur J Surg Oncol 2016;42:949-55.

25. Zinzindohoué C, Bertrand P, Michel A, et al. A Prospective Study on Skin-Sparing Mastectomy for Immediate Breast Reconstruction with Latissimus Dorsi Flap After Neoadjuvant Chemotherapy and Radiotherapy in Invasive 


\section{Page 10 of 10}

Breast Carcinoma. Ann Surg Oncol 2016;23:2350-6.

26. Lee JA, Yoon WS, Chung SY, et al. Can intensitymodulated radiation therapy spare the central flapped area while encompassing the target volume in radiotherapy after immediate breast reconstruction? J Med Imaging Radiat Oncol 2013;57:595-602.

27. Ohri N, Cordeiro PG, Keam J, et al. Quantifying the

Cite this article as: Yoon WS, Rim CH, Yang DS, Lee JA, Son GS, Chang YW, Woo SU, Kim DW, Dhong ES. Long-term outcomes of immediate autologous breast reconstruction after definite adjuvant therapy in intermediate and locally advanced breast cancer. Ann Transl Med 2019;7(23):743. doi: 10.21037/ atm.2019.11.129
Yoon et al. Long-term outcomes of breast reconstruction

impact of immediate reconstruction in postmastectomy radiation: a large, dose-volume histogram-based analysis. Int J Radiat Oncol Biol Phys 2012;84:e153-9.

28. Piper ML, Evangelista M, Amara D, et al. An Innovative Risk-Reducing Approach to Postmastectomy Radiation Delivery after Autologous Breast Reconstruction. Plast Reconstr Surg Glob Open 2017;5:e1265. 